\title{
Avaliação do nível de conhecimento de cirurgiões-dentistas e graduandos em Odontologia quanto ao manejo de indivíduos em uso de anticoagulantes orais
}

\author{
Assessment of dentists and dental students' level of knowledge in the management of individuals \\ using oral anticoagulants
}

\author{
Liciane dos Santos MENEZES ${ }^{a^{*}}$, Rosany Larissa Brito DE OLIVEIRA ${ }^{a}$, Luiz Carlos Ferreira DA SILVA ${ }^{a}$ \\ ${ }^{a} U F S$ - Universidade Federal de Sergipe, Aracaju, SE, Brasil
}

\begin{abstract}
Resumo
Introdução: Diante da alta incidência das doenças cardiovasculares, muitos são os pacientes que fazem uso de medicações para prevenção e tratamento dessas desordens. Consequentemente, o número de pessoas em uso de anticoagulantes orais a serem submetidas a tratamento odontológico é crescente e o manejo desses indivíduos requer criteriosa avaliação da condição clínica e do procedimento a ser realizado. Objetivo: Avaliar o nível de conhecimento de cirurgiões-dentistas e graduandos em Odontologia quanto ao manejo de indivíduos em uso de anticoagulantes orais a serem submetidos a tratamento odontológico. Material e método: Investigação descritiva, quantitativa, de corte transversal, com base em um questionário respondido por 100 indivíduos, sendo 70 cirurgiões-dentistas e 30 graduandos do curso de Odontologia, escolhidos por conveniência, para avaliar o conhecimento sobre o tema proposto. Para comparação das variáveis quantitativas, foi utilizado o Teste $t$ de student, para comparação de duas amostras, e o Teste ANOVA, com pós-teste de Bonferroni, para comparação de três amostras. Resultado: Dentre os entrevistados, $74,5 \%$ dos cirurgiões-dentistas e $54,2 \%$ dos graduandos relataram já ter atendido um paciente que faz uso de anticoagulante oral. Não houve diferença estatística quanto ao desempenho entre profissionais e alunos acerca do manejo odontológico de pacientes em uso de anticoagulantes. Dentre o grupo de cirurgiões-dentistas, os profissionais da cirurgia bucomaxilofacial e os que fizeram residência multiprofissional apresentaram melhor desempenho quando comparados aos clínicos gerais. Conclusão: Pôde-se concluir que, para os grupos estudados, o manejo de pessoas em uso de anticoagulantes orais ainda é um desafio.
\end{abstract}

Descritores: Anticoagulantes; inibidores da agregação de plaquetas; odontologia.

\begin{abstract}
Introduction: Cardiovascular diseases have a high incidence and due to that there is a large amount of patients who take medications for prevention and treatment of these disorders. Consequently, the number of people to be submitted to dental treatment and also under oral anticoagulant therapy is increasing and the management of these individuals requires a careful evaluation of the clinical condition and the procedure to be performed. Objective: To evaluate the level of knowledge of dentists and undergraduate dental students in the management of individuals using oral anticoagulants to be submitted to dental treatment. Material and method: Descriptive, quantitative, cross-sectional study based on a questionnaire answered by 100 individuals ( 70 dentists and 30 undergraduate dental students), chosen for convenience to evaluate their knowledge about the proposed topic. To compare the quantitative variables, the Student's T test was used to compare two samples and the ANOVA test with Bonferroni's post test to compare three samples. Result: Among the interviewees, $74.5 \%$ of dental surgeons and $54.2 \%$ of undergraduates reported having attended a patient under oral anticoagulant therapy. There was no statistical difference regarding the performance between professionals and students in the dental management of patients taking anticoagulants. Among the group of dental surgeons, the professionals of the bucomaxillofacial surgery and those who had multiprofessional residency presented better performance when compared to the general practitioners. Conclusion: The management of patients using oral anticoagulants stills a challenge for the groups studied.
\end{abstract}

Descriptors: Anticoagulants; platelet aggregation inhibitors; dentistry. 


\section{INTRODUÇÃO}

Diante do envelhecimento populacional e do aumento da expectativa de vida, a incidência de doenças crônicas, como cardiopatias e desordens vasculares, é cada vez mais comum ${ }^{1,2}$. De modo a prevenir futuros eventos tromboembólicos, o uso de antiagregantes plaquetários e anticoagulantes orais são amplamente utilizados na profilaxia e no tratamento destas doenças ${ }^{1-3}$. Consequentemente, o número de pessoas que necessitam de tratamento odontológico e fazem uso de anticoagulantes é crescente. Uma vez que essas medicações interferem no mecanismo da hemostasia, o risco de complicações hemorrágicas durante e após procedimentos odontológicos é uma preocupação para os cirurgiões-dentistas.

No passado, muitos dentistas indicavam a suspensão de anticoagulantes previamente a procedimentos odontológicos invasivos, a fim de reduzir o risco de sangramento ${ }^{3,4}$. Mas, atualmente, já se entende que, caso o procedimento odontológico a ser realizado seja considerado de menor magnitude e com baixo risco de sangramento, a suspensão dos anticoagulantes previamente ao procedimento é desnecessária ${ }^{2,4,5-7}$. Na maioria das vezes, o sangramento pós-operatório não é exclusivamente atribuído ao uso de antiagregantes plaquetários ${ }^{8}$ ou anticoagulantes orais ${ }^{2,5}$, quando medidas hemostáticas locais são corretamente adotadas. Fatores como dieta, uso concomitante de outros medicamentos e outras comorbidades podem alterar a atividade dos anticoagulantes ${ }^{5,9}$. Além disso, muitos estudos ressaltam que a alteração da dose ou a suspensão do anticoagulante oral previamente a procedimentos odontológicos de menor complexidade apresenta riscos de complicações tromboembólicas que sobrepõem os riscos de eventos hemorrágicos ${ }^{3-5,10-12}$.

Mesmo diante de alguns estudos na literatura, o tratamento odontológico de pessoas em terapia anticoagulante parece ainda ser um desafio para os cirurgiões-dentistas. Diante disto, este estudo tem como objetivo avaliar o nível de conhecimento de cirurgiões-dentistas e graduandos em Odontologia quanto ao manejo de usuários de anticoagulantes orais a serem submetidos a tratamento odontológico.

\section{MATERIAL E MÉTODO}

\section{População e Protocolo do Estudo}

O presente estudo foi aprovado pelo Comitê de Ética em Pesquisa da Universidade Federal de Sergipe e da Universidade Tiradentes, cumprindo todos os preceitos da resolução CNS 466/2012. Trata-se de uma investigação descritiva, quantitativa, de corte transversal, com base em um questionário preenchido por 100 indivíduos, sendo 70 cirurgiões-dentistas e 30 graduandos de Odontologia do Estado de Sergipe, Brasil.

O questionário foi previamente formulado pelos autores da pesquisa, contendo 11 perguntas fechadas, sendo três a respeito da experiência clínica e oito sobre o nível de conhecimento acerca do manejo de pessoas que fazem uso de anticoagulantes orais. Estas últimas oito perguntas foram baseadas em um caso clínico que considera um indivíduo do sexo masculino em uso contínuo de um anticoagulante oral, que necessita ser submetido a uma exodontia simples sob anestesia local. Os anticoagulantes em questão são aspirina, varfarina e dabigatrana.

Foi criado um score, que contemplava as questões de 4 a 7 , as quais avaliavam o nível de conhecimento sobre o manejo de pessoas em uso de anticoagulantes orais. Cada questão foi pontuada em zero $(0)$ ou 1 , em que 0 corresponde à resposta incorreta ou não tenho experiência ou conhecimento na área, e 1 contempla a resposta correta. Ao fim, obteve-se um score que variou de 0 a 8 , no qual 8 corresponde ao maior nível de conhecimento e 0 , ao menor.

Um estudo piloto foi realizado com cinco cirurgiões-dentistas e, de acordo com as suas sugestões, algumas perguntas foram modificadas e incluídas no questionário. No período de novembro a dezembro de 2017, cada indivíduo recebeu um envelope contendo um questionário autoaplicável e duas vias do Termo de Consentimento Livre e Esclarecido (TCLE), os quais foram posteriormente recolhidos pelo pesquisador.

Todos os participantes foram esclarecidos quanto aos objetivos da pesquisa e assinaram o Termo de Consentimento Livre e Esclarecido. Foram incluídos, no estudo, profissionais inscritos e registrados no Conselho Regional de Odontologia de Sergipe, e acadêmicos de graduação regularmente matriculados no último ano do curso de Odontologia. Questionários com respostas incompletas foram excluídos do estudo.

\section{Análise Estatística}

Os dados coletados foram tabulados numa planilha do programa Excel for Windows 2010 e posteriormente foram analisados no programa BioEstat (versão 5.3), sendo descritos em frequências absolutas, porcentagens, médias e desvio padrão.

Para testar a normalidade foi utilizado o Teste de Shapiro-Wilk, pelo qual se verificou que os dados das variáveis estudadas seguem uma distribuição normal.

Para comparação das variáveis quantitativas, foi utilizado o Teste $t$ de student, para comparação de duas amostras, e o teste ANOVA, com pós-teste de Bonferroni, para comparação de três amostras. O nível de significância adotado foi igual a 5\%; portanto, se o p-valor for menor que o nível de significância $(p<0,05)$, rejeita-se a hipótese nula, isto é, existem evidências de que os grupos diferem.

\section{RESULTADO}

Dentre os 100 questionários aplicados, 78 (78\%) foram elegíveis para análise estatística, sendo $55(78,6 \%)$ do grupo de cirurgiões-dentistas e $23(76,6 \%)$ do grupo de graduandos. Os questionários que se apresentavam parcialmente respondidos foram excluídos.

Na Tabela 1, pode ser observado o perfil de experiência clínica dos participantes, em que a maioria dos cirurgiões-dentistas $(70,9 \%)$ e graduandos $(95,6 \%)$ realiza procedimentos odontológicos invasivos em sua rotina clínica. A maioria também já atendeu algum indivíduo que faz uso de anticoagulante oral, sendo 74,5\% dos cirurgiões-dentistas e $54,2 \%$ dos graduandos. Entretanto, a maioria deles relatou não ter vivenciado algum caso de sangramento 
excessivo pós-operatório em sua rotina clínica, sendo 63,6\% dos cirurgiões-dentistas e 91,3\% dos graduandos.

A Tabela 2 compreende as perguntas de 4 a 7 , que avaliam o nível de conhecimento dos participantes acerca do manejo odontológico de pessoas que fazem uso de anticoagulantes orais. Foi observado que a maioria dos participantes dessa pesquisa, tanto do grupo de cirurgiões-dentistas quanto do grupo de graduandos, apresenta um baixo nível de conhecimento ou não tem experiência ou conhecimento ao que lhes foi questionado.

Na Tabela 3, foi avaliado se os participantes que relataram, na sua rotina clínica, já ter ocorrido algum caso de sangramento excessivo pós-operatório, saberiam conduzir corretamente essa complicação. Observou-se que grande parte dos participantes não está preparada, uma vez que a maioria escolheu uma alternativa incoerente para o manejo desse evento.

Na Figura 1, foi avaliado o nível de conhecimento nas diferentes especialidades e pôde-se observar que os cirurgiões-dentistas que pertenciam ao grupo de cirurgiões bucomaxilofaciais ou da residência multiprofissional apresentaram um maior nível de conhecimento, quando comparados aos cirurgiões-dentistas clínicos gerais.

Comparou-se também o nível de conhecimento por tempo de formação dos cirurgiões-dentistas e observou-se que não houve diferença estatística significativa [Teste $t$ de student ( $\mathrm{p}$-valor $=0,9$ )]. Do mesmo modo, também não houve diferença estatística significativa quando foi comparado o nível de conhecimento dos cirurgiões-dentistas e dos graduandos de Odontologia [Teste $t$ de student $(\mathrm{p}$-valor $=0,7)$.

Tabela 1. Estatística descritiva da amostra de profissionais e graduandos de Odontologia - Perguntas de 1 a 3

\begin{tabular}{|c|c|c|c|c|}
\hline \multirow[b]{2}{*}{ Perguntas } & \multicolumn{2}{|c|}{ Cirurgião-dentista } & \multicolumn{2}{|c|}{ Graduando de Odontologia } \\
\hline & $\begin{array}{c}\text { Sim } \\
\text { n }(\%)\end{array}$ & $\begin{array}{c}\text { Não } \\
\text { n (\%) }\end{array}$ & $\begin{array}{c}\text { Sim } \\
\text { n }(\%)\end{array}$ & $\begin{array}{l}\text { Não } \\
\text { n (\%) }\end{array}$ \\
\hline $\begin{array}{l}1 \text { Você realiza procedimentos, como exodontia, biópsia } \\
\text { e/ou raspagem subgengival, em sua rotina clínica? }\end{array}$ & $\begin{array}{c}39 \\
(70,9 \%)\end{array}$ & $\begin{array}{c}16 \\
(29,1 \%)\end{array}$ & $\begin{array}{c}22 \\
(95,6 \%)\end{array}$ & $\begin{array}{c}1 \\
(4,4 \%)\end{array}$ \\
\hline $\begin{array}{l}3 \mathrm{Na} \text { sua rotina clínica já ocorreu algum caso de } \\
\text { sangramento excessivo pós-operatório? }\end{array}$ & $\begin{array}{c}20 \\
(36,4 \%)\end{array}$ & $\begin{array}{c}35 \\
(63,6 \%)\end{array}$ & $\begin{array}{c}2 \\
(8,7 \%)\end{array}$ & $\begin{array}{c}21 \\
(91,3 \%)\end{array}$ \\
\hline
\end{tabular}

Tabela 2. Estatística descritiva da amostra de profissionais e graduandos de Odontologia - Perguntas de 4 a 7

\begin{tabular}{|c|c|c|c|c|c|c|}
\hline \multirow[b]{2}{*}{ Perguntas } & \multicolumn{3}{|c|}{ Cirurgião-dentista } & \multicolumn{3}{|c|}{ Graduando de Odontologia } \\
\hline & $\begin{array}{l}\text { Certo } \\
\text { n (\%) }\end{array}$ & $\begin{array}{c}\text { Errado } \\
\text { n (\%) }\end{array}$ & $\begin{array}{c}\text { Sem } \\
\text { experiência } \\
\text { ou } \\
\text { conhecimento } \\
\text { n (\%) }\end{array}$ & $\begin{array}{l}\text { Certo } \\
\text { n (\%) }\end{array}$ & $\begin{array}{c}\text { Errado } \\
\text { n (\%) }\end{array}$ & $\begin{array}{c}\text { Sem } \\
\text { experiência } \\
\text { ou } \\
\text { conhecimento } \\
\text { n (\%) }\end{array}$ \\
\hline
\end{tabular}

4 Considerando que o paciente faz uso continuado de aspirina (um antiagregante plaquetário), na dose de $100 \mathrm{mg}$, uma vez ao dia:

4.1 Qual a sua conduta de

rotina para pacientes que

fazem uso de antiagregantes

$24(43,6 \%) \quad 31(56,4 \%)$

$10(43,5 \%)$

$13(56,5 \%)$

plaquetários?

4.2 Em caso de solicitação de exames laboratoriais, qual o parâmetro laboratorial você adotaria para sua tomada de decisão clínica?

\begin{tabular}{|c|c|c|c|c|c|}
\hline $\begin{array}{c}18 \\
(32,7 \%)\end{array}$ & $24(43,6 \%)$ & $\begin{array}{c}13 \\
(23,7 \%)\end{array}$ & $\begin{array}{c}6 \\
(26,1 \%)\end{array}$ & $14(60,9 \%)$ & $\begin{array}{c}3 \\
(13,0 \%)\end{array}$ \\
\hline
\end{tabular}

5 Caso o paciente faça uso continuado da varfarina (anticoagulante oral - antagonista da vitamina K), na dose de 2,5 mg, uma vez ao dia, responda:

$\begin{array}{lccc}5.1 \text { Qual a sua conduta } & 18 & 37(67,3 \%) & -16(69,6 \%) \\ \text { rotineira para pacientes nessa } & (32,7 \%) & & (30,4 \%)\end{array}$

\begin{tabular}{|c|c|c|c|c|c|c|}
\hline $\begin{array}{l}5.2 \text { Em caso de solicitação } \\
\text { de exames laboratoriais, } \\
\text { qual período prévio } \\
\text { ao procedimento você } \\
\text { rotineiramente os solicitaria? }\end{array}$ & $\begin{array}{c}14 \\
(25,5 \%)\end{array}$ & $\begin{array}{c}18 \\
(32,7 \%)\end{array}$ & $\begin{array}{c}23 \\
(41,8 \%)\end{array}$ & $\begin{array}{c}5 \\
(21,7 \%)\end{array}$ & $\begin{array}{c}5 \\
(21,7 \%)\end{array}$ & $\begin{array}{c}13 \\
(56,6 \%)\end{array}$ \\
\hline $\begin{array}{l}\text { 5.3 Qual parâmetro laboratorial } \\
\text { você utilizaria para sua tomada } \\
\text { de decisão clínica? }\end{array}$ & $16(29,1 \%)$ & $18(32,7 \%)$ & $\begin{array}{c}21 \\
(38,2 \%)\end{array}$ & $3(13,0 \%)$ & $6(26,1 \%)$ & $\begin{array}{c}14 \\
(60,9 \%)\end{array}$ \\
\hline
\end{tabular}


Tabela 2. Continuação...

\begin{tabular}{|c|c|c|c|c|c|c|}
\hline \multirow[b]{2}{*}{ Perguntas } & \multicolumn{3}{|c|}{ Cirurgião-dentista } & \multicolumn{3}{|c|}{ Graduando de Odontologia } \\
\hline & $\begin{array}{l}\text { Certo } \\
\text { n (\%) }\end{array}$ & $\begin{array}{c}\text { Errado } \\
\text { n (\%) }\end{array}$ & $\begin{array}{c}\text { Sem } \\
\text { experiência } \\
\text { ou } \\
\text { conhecimento } \\
\text { n (\%) }\end{array}$ & $\begin{array}{l}\text { Certo } \\
\text { n (\%) }\end{array}$ & $\begin{array}{c}\text { Errado } \\
\text { n (\%) }\end{array}$ & $\begin{array}{c}\text { Sem } \\
\text { experiência } \\
\text { ou } \\
\text { conhecimento } \\
\text { n (\%) }\end{array}$ \\
\hline \multicolumn{7}{|c|}{$\begin{array}{l}6 \text { Ao longo da última década, foram desenvolvidos novos anticoagulantes orais (inibidores do fator Xa e inibidores diretos da trombina) com } \\
\text { características de eficácia e segurança importantes em relação ao tratamento convencional. Uma dessas novas drogas é a dabigatrana (Pradaxa), um } \\
\text { inibidor direto da trombina ou fator II. Caso o paciente descrito faça uso deste anticoagulante na posologia de } 110 \mathrm{mg} \text {, duas vezes ao dia, responda: }\end{array}$} \\
\hline $\begin{array}{l}6.1 \text { Qual a sua conduta de } \\
\text { rotina para pacientes nessa } \\
\text { condição clínica? }\end{array}$ & $\begin{array}{c}14 \\
(25,5 \%)\end{array}$ & $\begin{array}{c}31 \\
(56,4 \%)\end{array}$ & $\begin{array}{c}10 \\
(18,1 \%)\end{array}$ & $\begin{array}{c}11 \\
(47,8 \%)\end{array}$ & $\begin{array}{c}9 \\
(39,2 \%)\end{array}$ & $\begin{array}{c}3 \\
(13,0 \%)\end{array}$ \\
\hline $\begin{array}{l}6.2 \text { Em caso de solicitação de } \\
\text { exames laboratoriais, qual o } \\
\text { parâmetro laboratorial você } \\
\text { adotaria para sua tomada de } \\
\text { decisão clínica? }\end{array}$ & $\begin{array}{c}2 \\
(3,6 \%)\end{array}$ & $\begin{array}{c}15 \\
(27,3 \%)\end{array}$ & $\begin{array}{c}38 \\
(69,1 \%)\end{array}$ & $\begin{array}{c}1 \\
(4,4 \%)\end{array}$ & $\begin{array}{c}7 \\
(30,4 \%)\end{array}$ & $\begin{array}{c}15 \\
(65,2 \%)\end{array}$ \\
\hline $\begin{array}{l}7 \text { Em uma situação de possível } \\
\text { sangramento excessivo } \\
\text { pós-operatório, quais medidas } \\
\text { você adotaria para controle } \\
\text { desse sangramento? }\end{array}$ & $\begin{array}{c}22 \\
(40,0 \%)\end{array}$ & $\begin{array}{c}2 \\
(3,6 \%)\end{array}$ & $\begin{array}{c}31 \\
(56,4 \%)\end{array}$ & $\begin{array}{c}11 \\
(47,8 \%)\end{array}$ & $\begin{array}{c}5 \\
(21,7 \%)\end{array}$ & $\begin{array}{c}7 \\
(30,5 \%)\end{array}$ \\
\hline
\end{tabular}

Tabela 3. Estatística descritiva dos profissionais e estudantes de Odontologia que relataram, na sua rotina clínica, já ter ocorrido algum caso de sangramento excessivo pós-operatório - Pergunta 7

\begin{tabular}{|c|c|c|c|c|c|c|}
\hline \multirow[b]{2}{*}{ Perguntas } & \multicolumn{3}{|c|}{ Cirurgião-dentista } & \multicolumn{3}{|c|}{ Graduando de Odontologia } \\
\hline & $\begin{array}{l}\text { Certo } \\
\text { n (\%) }\end{array}$ & $\begin{array}{c}\text { Errado } \\
\text { n (\%) }\end{array}$ & $\begin{array}{c}\text { Sem } \\
\text { experiência } \\
\text { ou } \\
\text { conhecimento } \\
\text { n (\%) }\end{array}$ & $\begin{array}{l}\text { Certo } \\
\text { n (\%) }\end{array}$ & $\begin{array}{c}\text { Errado } \\
\text { n (\%) }\end{array}$ & $\begin{array}{c}\text { Sem } \\
\text { experiência } \\
\text { ou } \\
\text { conhecimento } \\
\text { n (\%) }\end{array}$ \\
\hline $\begin{array}{l}7 \text { Em uma situação de possível } \\
\text { sangramento excessivo } \\
\text { pós-operatório, quais medidas } \\
\text { você adotaria para controle } \\
\text { desse sangramento? }\end{array}$ & $\begin{array}{c}7 \\
(35,0 \%)\end{array}$ & $\begin{array}{c}9 \\
(45,0 \%)\end{array}$ & $\begin{array}{c}4 \\
(20,0 \%)\end{array}$ & $\begin{array}{c}0 \\
(0,0 \%)\end{array}$ & $\begin{array}{c}2 \\
(100,0 \%)\end{array}$ & $\begin{array}{c}0 \\
(0,0 \%)\end{array}$ \\
\hline
\end{tabular}

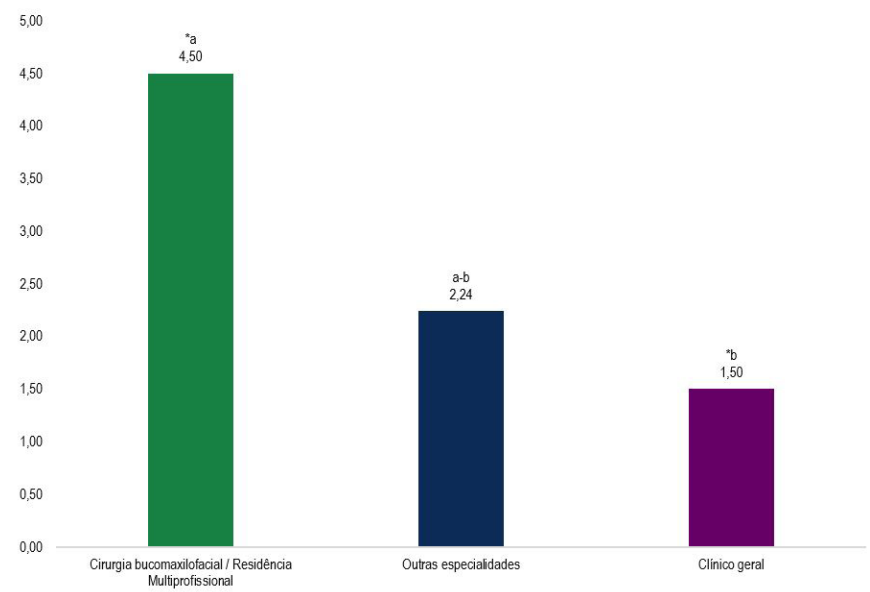

Figura 1. Avaliação do nível de conhecimento nas diferentes especialidades. ANOVA, pós teste de Bonferroni; ${ }^{*}$ Letras diferentes indicam diferenças estatísticas significativa entre os grupos ( $\mathrm{p}$-valor $<0,05)$.

\section{DISCUSSÃO}

Este estudo teve como objetivo avaliar o nível de conhecimento de cirurgiões-dentistas e graduandos em Odontologia do Estado de Sergipe quanto ao manejo de pessoas em uso de anticoagulantes orais, que necessitam ser submetidas a tratamento odontológico.

Sabe-se que questionários aplicados de forma presencial apresentam um índice maior de resposta, quando comparados aos questionários eletrônicos ou enviados por correio ${ }^{13,14}$. Entretanto, os questionários respondidos com a presença de um entrevistador podem apresentar resultados tendenciosos ${ }^{4}$. Dessa forma, na presente pesquisa, os questionários foram respondidos sem a presença de um entrevistador. Os mesmos foram entregues individualmente, mas, num segundo momento, os envelopes foram coletados, preservando, assim, a identidade dos participantes. 
Apesar da importância do tema, este estudo apresenta algumas limitações, sobretudo quanto à pequena amostra de participantes, que se deu em função de $22 \%$ dos questionários terem sido excluídos por apresentarem questões não respondidas.

Os anticoagulantes orais utilizados como instrumento de estudo, nesta pesquisa, foram aspirina, varfarina e dabigatrana. A aspirina e a varfarina são os mais utilizados dentro de suas respectivas categorias, sendo consideradas drogas de baixo custo e alta confiabilidade ${ }^{1,15}$. Já a dabigatrana foi o primeiro novo anticoagulante introduzido no mercado para uso em seres humanos ${ }^{16}$ e possui vantagens clínicas, como não necessitar de exames laboratoriais de rotina para monitoração da coagulação e a existência de um antídoto, o idarucizumab, em caso de sangramento prolongado e incontrolável. Além disso, possui rápido início de ação, meia vida curta, alta margem terapêutica, baixa interação com outras drogas e quase nenhuma interação alimentar, podendo ser uma excelente alternativa à varfarina ${ }^{6}$. No entanto, o seu alto custo pode ser considerado uma limitação ${ }^{1}$.

A maioria dos participantes (70,9\% dos cirurgiões-dentistas e $95,6 \%$ dos graduandos) relatou realizar rotineiramente procedimentos odontológicos invasivos, os quais, em sua maioria, apresentam algum risco de sangramento. Esses procedimentos podem variar desde uma raspagem supragengival a procedimentos mais extensos, como extração dentária, raspagem subgengival e biópsia ${ }^{5,12}$. Independentemente da magnitude do procedimento a ser executado, é sempre importante analisá-lo e correlacioná-lo à condição clínica do indivíduo que faz uso de um anticoagulante oral, a fim de que não sejam tomadas decisões que resultem em eventos infortúnios.

Nesta pesquisa, a maioria dos participantes avaliados relatou já ter atendido algum indivíduo que faz uso de anticoagulante oral, correspondendo a $74,5 \%$ dos cirurgiões-dentistas e $54,2 \%$ dos graduandos. O número de pessoas em uso de anticoagulantes orais a serem submetidos a tratamento odontológicos é crescente, visto que as doenças cardiovasculares são as principais causas de morte no Brasil ${ }^{17}$. Verificou-se, ainda, na presente pesquisa, que apenas uma minoria dos entrevistados já vivenciou algum evento de sangramento excessivo pós-operatório em sua rotina clínica, corroborando com alguns estudos que mostram que a incidência de sangramento excessivo pós-operatório em pessoas que fazem uso de anticoagulantes orais é baixa ${ }^{2,12,15,18-21}$. Apenas 20 a $25 \%$ dos indivíduos que usam aspirina apresentam tempo de sangramento anormal $^{22}$. Evidências mostram que estes medicamentos podem dobrar o tempo de sangramento basal e, mesmo assim, apresentar valores dentro da normalidade. Dessa forma, observa-se que, uma vez que a faixa terapêutica dos anticoagulantes orais esteja estável, raramente ocorrerá um evento hemorrágico ${ }^{23}$.

Além disso, as complicações hemorrágicas podem ser controladas ou evitadas com o uso de manobras e agentes hemostáticos locais, e, dentre os mais utilizados, figuram a sutura, a compressa com gaze sobre o sítio cirúrgico, a esponja de gelatina, os bochechos com ácido tranexâmico e o selante de fibrina ${ }^{2,9,12,15,18,19,21}$.

Verificou-se, ainda, que a maioria dos participantes apresentou um baixo nível de conhecimento quanto à solicitação de exames laboratoriais, para verificação do risco de sangramento de indivíduos em uso de aspirina.

Sabe-se que, dentre os parâmetros laboratoriais de escolha para avaliação de risco de sangramento em pessoas que utilizam a aspirina, os mais apropriados são o tempo de sangramento (TS) e o teste de análise da função plaquetária (PFA-100). Atualmente, a literatura reforça que apesar de o TS (método de Ivy ou Duke) ser mais acessível, não é o mais sensível e não prediz o aumento de perda de sangue em cirurgias odontológicas ${ }^{18}$; assim, o PFA-100 seria mais específico e menos invasivo para a mensuração da função plaquetária ${ }^{9}$. No entanto, o uso do PFA-100 ainda não está amplamente difundido no Brasil ${ }^{24}$. Dessa forma, nesta pesquisa, ambos os testes foram considerados opções acertadas, ou seja, foram considerados como resposta correta, tanto a mensuração do TS quanto o PFA-100, para monitoração do risco de sangramento da aspirina.

Quanto ao manejo dos indivíduos que fazem uso de anticoagulantes orais antagonistas da vitamina $\mathrm{K}$, a conduta considerada como mais apropriada é atender normalmente o indivíduo e solicitar exames laboratoriais. A fim de monitorar a ação da varfarina, o parâmetro laboratorial mais relevante é a Relação Normatizada Internacional (INR), que foi introduzido em 1983 pela Organização Mundial de Saúde com o intuito de obter o tempo de protrombina corrigido segundo padrões mundiais 9 .

Aspectos como a resposta individual de cada pessoa, a dieta ou a administração simultânea de outras drogas podem alterar a atividade da varfarina, necessitando assim de uma monitoração frequente do seu nível de anticoagulação ${ }^{5,9,25}$. Assim, a avaliação da faixa terapêutica da varfarina deve ser idealmente avaliada no mesmo dia do procedimento odontológico, em especial para pessoas com coagulação instável. Já para aqueles em situação estável, a monitoração pode ser feita em até três dias antes do procedimento $^{19,25}$. No nosso questionário, foram consideradas ambas as opções de conduta como resposta correta (aferição no mesmo dia e três dias antes da realização do procedimento), uma vez que não estava especificado se o indivíduo encontrava-se com anticoagulação estável ou instável.

Diante de algumas dificuldades práticas no manejo de pessoas que fazem uso dos antagonistas da vitamina $\mathrm{K}$, novos anticoagulantes orais foram introduzidos. O primeiro a ser aprovado para uso em seres humanos foi a dabigatrana ${ }^{16}$, um inibidor direto da trombina, que fornece uma anticoagulação estável sem necessidade de monitoração de rotina da coagulação ${ }^{1,5,6}$. Quando necessária, a aferição do risco de sangramento da dabigatrana pode ser realizada pelo tempo de trombina (TT) ou pelo tempo de coagulação de Ecarin (ECT), que é ainda mais sensível; no entanto, este não é facilmente disponível na clínica ${ }^{5}$. Portanto, a dabigatrana é considerada uma droga bastante segura, sendo a sua monitoração geralmente desnecessária, exceto em casos de cirurgia de emergência ou hemorragias ${ }^{6}$.

Ao avaliar a conduta dos cirurgiões-dentistas e graduandos acerca de pessoas que fazem uso de anticoagulantes orais, foi observado, neste estudo, que os participantes ainda não estão bem preparados para lidar com esse perfil de pessoas, inclusive os que afirmaram já ter tido experiência com esses indivíduos, uma vez que apresentaram condutas equivocadas ou relataram não ter conhecimento sobre o manejo. Tais resultados corroboram com 
alguns estudos realizados anteriormente que concluíram ainda existir uma abordagem clínica fragilizada e inconsistente por parte dos cirurgiões-dentistas a esse grupo de pessoas ${ }^{4,19,25}$.

Em estudos realizados por Chinnaswami et al. ${ }^{4} \mathrm{e}$ Dewan et al. ${ }^{25}$, foi descrito o tempo de formado dos indivíduos entrevistados, mas isto não foi comparado ao desempenho obtido no questionário. Em nossa pesquisa, o tempo médio de formado dos cirurgiões-dentistas foi de 7,5 anos. Ao se avaliar o nível de conhecimento por tempo de formação, não se observou diferença estatística significativa entre profissionais recém-formados e mais experientes.

Não foram observados relatos na literatura da aplicação deste tipo de questionário em graduandos, portanto foi decidida a inclusão deste grupo, o qual cursava o último ano de Odontologia, uma vez que, em breve, serão profissionais e deverão estar providos de embasamento científico e preparados para o atendimento a essa população.

Quando foi avaliado o nível de conhecimento por especialidade odontológica, pôde-se observar que somente houve diferença estatística significativa em relação ao grupo clínico geral quando este foi comparado ao grupo cirurgia bucomaxilofacial/residência multiprofissional. Acredita-se que o melhor desempenho deste grupo está relacionado à maior vivência desses profissionais em ambiente hospitalar, pois assim possuem mais contato com pessoas de maior complexidade sistêmica ${ }^{3}$.

\section{CONCLUSÃO}

Por fim, pôde-se observar que a maioria dos participantes apresentou um baixo nível de conhecimento quanto à abordagem de pessoas que fazem uso de anticoagulantes orais, uma vez que apresentaram condutas equivocadas ou relataram não ter conhecimento ou experiência sobre esse manejo. Dessa forma, conclui-se que essa temática ainda é um desafio para a classe odontológica e que a conscientização desse conhecimento e cuidado desde a graduação é primordial para o exercício de uma Odontologia com maior embasamento e segurança.

\section{REFERÊNCIAS}

1. Mingarro-de-León A, Chaveli-López B. Alternative to oral dicoumarin anticoagulants: considerations in dental care. J Clin Exp Dent. 2013 Dec 1;5(5):e273-8. http://dx.doi.org/10.4317/jced.51226. PMid: 24455094.

2. Shi Q, Xu J, Zhang T, Zhang B, Liu H. Post-operative bleeding risk in dental surgery for patients on oral anticoagulant therapy: a metaanalysis of observational studies. Front Pharmacol. 2017 Feb 8;8:58. http://dx.doi.org/10.3389/fphar.2017.00058. PMid: 28228727.

3. van Diermen DE, van der Waal I, Hoogvliets MW, Ong FN, Hoogstraten J. Survey response of oral and maxillofacial surgeons on invasive procedures in patients using antithrombotic medication. Int J Oral Maxillofac Surg. 2013 Apr;42(4):502-7. http://dx.doi.org/10.1016/j. ijom.2012.09.018. PMid:23123097.

4. Chinnaswami R, Bagadia RK, Mohan A, Kandaswamy E, Chandrasekaran D. Dentists' knowledge, attitude and practice in treating patients taking oral antithrombotic medications - a survey. J Clin Diagn Res. 2017 Jan;11(1):ZC88-91. http://dx.doi.org/10.7860/JCDR/2017/23648.9236. PMid:28274053.

5. Hong CH, Islam I. Anti-thrombotic therapy: implications for invasive outpatient procedures in dentistry. J Blood Disord Transfus. $2013 ; 4: 166$. http://dx.doi.org/10.4172/2155-9864.1000166.

6. Costantinides F, Rizzo R, Pascazio L, Maglione M. Managing patients taking novel oral anticoagulants (NOAs) in dentistry: a discussion paper on clinical implications. BMC Oral Health. 2016 Jan;16(1):5. http://dx.doi.org/10.1186/s12903-016-0170-7. PMid:26822674.

7. Curto A, Curto D, Sanchez J. Managing patients taking edoxaban in dentistry. J Clin Exp Dent. 2017 Feb 1;9(2):e308-e311. http://dx.doi. org/10.4317/jced.53431. PMid: 28210454.

8. Rai R, Mohan B, Babbar V, Dang N. Practices and perceptions of doctors for patients on anti-platelets during dental surgery: a national survey. J Maxillofac Oral Surg. 2014 Sep;13(3):249-52. http://dx.doi.org/10.1007/s12663-013-0523-6. PMid:25018596.

9. Little JW, Miller CS, Henry RG, McIntosh BA. Antithrombotic agents: implications in dentistry. Oral Surg Oral Med Oral Pathol Oral Radiol Endod. 2002 May;93(5):544-51. http://dx.doi.org/10.1067/moe.2002.121391. PMid:12075203.

10. Campbell JH, Alvarado F, Murray RA. Anticoagulation and minor oral surgery: should the anticoagulation regimen be altered? J Oral Maxillofac Surg. 2000 Feb;58(2):131-5, discussion 135-6. http://dx.doi.org/10.1016/S0278-2391(00)90324-0. PMid:10670590.

11. Beirne OR. Evidence to continue oral anticoagulant therapy for ambulatory oral surgery. J Oral Maxillofac Surg. 2005 Apr;63(4):540-5. http://dx.doi.org/10.1016/j.joms.2004.12.009. PMid:15789328.

12. Dézsi CA, Dézsi BB, Dézsi AD. Management of dental patients receiving antiplatelet therapy or chronic oral anticoagulation: a review of the latest evidence. Eur J Gen Pract. 2017 Dec;23(1):196-201. http://dx.doi.org/10.1080/13814788.2017.1350645. PMid:28743214.

13. Cartwright A. Professionals as responders: variations in and effects of response rates to questionnaires, 1961-77. BMJ. 1978 Nov;2(6149):141921. http://dx.doi.org/10.1136/bmj.2.6149.1419. PMid:719433.

14. Brazier JE, Harper R, Jones NM, O'Cathain A, Thomas KJ, Usherwood T, et al. Validating the SF-36 health survey questionnaire: new outcome measure for primary care. BMJ. 1992 Jul;305(6846):160-4. http://dx.doi.org/10.1136/bmj.305.6846.160. PMid:1285753.

15. Krishnan B, Shenoy NA, Alexander M. Exodontia and antiplatelet therapy. J Oral Maxillofac Surg. 2008 Oct;66(10):2063-6. http://dx.doi. org/10.1016/j.joms.2008.06.027. PMid:18848103.

16. Mekaj YH, Mekaj AY, Duci SB, Miftari EI. New oral anticoagulants: their advantages and disadvantages compared with vitamin K antagonists in the prevention and treatment of patients with thromboembolic events. Ther Clin Risk Manag. 2015 Jun;11:967-77. http://dx.doi.org/10.2147/ TCRM.S84210. PMid:26150723. 
17. Mansur AP, Favarato D. Trends in mortality rate from cardiovascular disease in Brazil, 1980-2012. Arq Bras Cardiol. 2016 Jul;107(1):20-5. http://dx.doi.org/10.5935/abc.20160077. PMid:27223642.

18. Brennan MT, Wynn RL, Miller CS. Aspirin and bleeding in dentistry: an update and recommendations. Oral Surg Oral Med Oral Pathol Oral Radiol Endod. 2007 Sep;104(3):316-23. http://dx.doi.org/10.1016/j.tripleo.2007.03.003. PMid:17618144.

19. Murphy J, Twohig E, McWilliams SR. Dentists' approach to patients on anti-platelet agents and warfarin: a survey of practice. J Ir Dent Assoc. 2010 Feb-Mar;56(1):28-31. PMid:20337143.

20. Sáez-Alcaide LM, Sola-Martín C, Molinero-Mourelle P, Paredes-Rodríguez V, Zarrias-Caballero C, Hernández-Vallejo G. Dental management in patients with antiplatelet therapy: a systematic review. J Clin Exp Dent. 2017 Aug 1;9(8):e1044-e1050. http://dx.doi.org/10.4317/jced.54079. PMid: 28936297.

21. Dantas AK, Deboni MCZ, Piratininga JL. Cirurgias odontológicas em usuários de anticoagulantes orais. Rev Bras Hematol Hemoter. 2009 Jan 21;31(5):337-40. http://dx.doi.org/10.1590/S1516-84842009005000071.

22. Owens CD, Belkin M. Thrombosis and coagulation: operative management of the anticoagulated patient. Surg Clin North Am. 2005 Dec;85(6):1179-89, x. http://dx.doi.org/10.1016/j.suc.2005.09.008. PMid:16326201.

23. Blinder D, Manor Y, Martinowitz U, Taicher S. Dental extractions in patients maintained on oral anticoagulant therapy: comparison of INR value with occurrence of postoperative bleeding. Int J Oral Maxillofac Surg. 2001 Dec;30(6):518-21. http://dx.doi.org/10.1054/ijom.2001.0172. PMid:11829234.

24. Hoffbrand AV, Moss PAH. Fundamentos em hematologia de Hoffbrand. 7 ed. Porto Alegre: Artmed; 2018.

25. Dewan K, Bishop K, Muthukrishnan A. Management of patients on warfarin by general dental practitioners in South West Wales: continuing the audit cycle. Br Dent J. 2009 Feb 28;206(4):E8; discussion 214-5. http://dx.doi.org/10.1038/sj.bdj.2009.112.

\section{CONFLITOS DE INTERESSE}

Os autores declaram não haver conflitos de interesse.

\section{*AUTOR PARA CORRESPONDÊNCIA}

Liciane dos Santos Menezes, Av. Deputado Silvio Teixeira, 1333, 402B, Bairro Jardins, 49025-100 Aracaju - SE, Brasil, e-mail: licianesmenezes@gmail.com

Recebido: Setembro 26, 2018

Aprovado: Outubro 19, 2018 